\title{
Oxidation of primary and secondary alcohols by 4-acetylamino- 2,2,6,6-tetramethylpiperidine-1-oxoammonium tetrafluoroborate in aqueous media
}

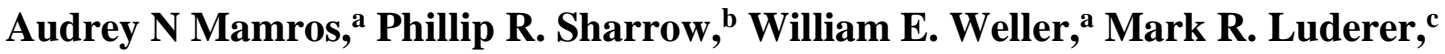 \\ Justin D. Fair, ${ }^{\text {b }}$ Kristina O. Pazehoski, ${ }^{\text {a }}$ and Matthew R. Luderer ${ }^{a *}$ \\ ${ }^{a}$ Department of Natural Science, Engineering, and Mathematics, University of Pittsburgh \\ at Greensburg, Greensburg PA, 15601, USA \\ ${ }^{b}$ Department of Chemistry, Indiana University of Pennsylvania, Indiana, PA, 15701, USA \\ ${ }^{c}$ Department of Chemistry, University of Connecticut, Storrs, CT, 06269, USA \\ E-mail: mrl27@pitt.edu
}

Dedicated to William F. Bailey on the occasion of his $65^{\text {th }}$ birthday

DOI: $\underline{\text { http://dx.doi.org/10.3998/ark.5550190.0012.504 }}$

\begin{abstract}
The water soluble oxidant 4-acetylamino-2,2,6,6-tetramethylpiperidine-1-oxoammonium tetrafluoroborate 1 oxidizes primary and secondary aliphatic, primary allylic, and primary and secondary benzylic alcohols to their corresponding aldehydes and ketones in aqueous media in good to excellent yields.
\end{abstract}

Keywords: Oxoammonium salt, oxidation, aqueous organic chemistry

\section{Introduction}

The use of nitroxides and oxoammonium salts as potent and selective oxidative methods in organic synthesis has found widespread applications as early as the 1960s. ${ }^{1}$ More specifically, oxoammonium salts such as $\mathbf{1}$ (Figure 1), as well as those salts composed of diverse anions (e.g. perchlorates, chlorides, etc.), ${ }^{2}$ have been employed in common organic solvents for the oxidation of primary alcohols to aldehydes and secondary alcohols to ketones with or devoid of alumina or silica gel as catalyst. ${ }^{3}$ Although there have been reports ${ }^{4}$ that mention the potential risk for the perchlorate salt to detonate upon heating after long term storage, most current methods rely on the more attractive tetrafluoroborate oxoammonium salt due to its lasting stability. ${ }^{5}$ 


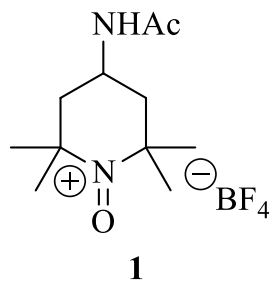

Figure 1. 4-Acetylamino-2,2,6,6-tetramethylpiperidine-1-oxoammonium tetrafluoroborate.

Potentially carcinogenic organic solvent systems such as methylene chloride or a 1:1 mixture of ethyl acetate-methylene chloride seem to be the preferred choice for oxoammonium salt oxidations as these solvent systems have been shown to afford highly pure carbonyls products in excellent yield. ${ }^{3}$ In recent years, the use of aqueous solvent systems in organic synthesis has attracted considerable attention within the chemical community; as water is inexpensive, readily available, easy to purify, and environmentally benign. ${ }^{6}$ The replacement of traditional organic solvents with aqueous systems may provide alternative modes of reactivity. ${ }^{5}$ For example, Diels-Alder type reactions have been found to be greatly accelerated under these conditions as it has been suggested to undergo the hydrophobic effect. ${ }^{6 a, 7}$

The toxicity of the traditional solvent systems as well as the recent interest in aqueous promoted media prompted the study of the aqueous oxidation of alcohols with $\mathbf{1}$. Indeed, a diverse group of primary and secondary aliphatic, primary allylic, and primary and secondary benzylic alcohols can be oxidized in the presence of $\mathbf{1}$ with and without catalyst (silica gel or alumina) in an aqueous media.

\section{Results and Discussion}

Initial experiments to optimize the reaction conditions for the oxidation of alcohols to their corresponding carbonyls were performed with benzyl alcohol as substrate in the presence of various amounts of $\mathbf{1}$, added catalyst, ${ }^{2 \mathrm{f}, 6 \mathrm{~b}}$ time, and temperature. The results of this part of the study are summarized in Table 1. The conversion of benzyl alcohol to benzaldehyde was quite facile employing 1.1 molar equiv of $\mathbf{1}$ for six hours at reflux (Table 1, entry 8). The addition of a catalyst was found to have a negligible effect (Table 1, entries 9 and 10). Increasing the molar equiv of 1 resulted in over oxidation to afford benzoic acid (Table 1, entries 11 and 12). 
Table 1. Optimization of the reaction conditions for the conversion of benzyl alcohol to benzaldehyde using $\mathbf{1}$ in aqueous media.

\begin{tabular}{|c|c|c|c|c|c|}
\hline Entry & Mole equiv $\mathbf{1}$ & Temp $\left({ }^{\circ} \mathrm{C}\right)$ & Time (h) & Catalyst & $\%$ Yield $^{\mathrm{a}}$ \\
\hline 1 & 1.0 & 21 & 24 & None & 80 \\
\hline 2 & 1.1 & 21 & 24 & None & 87 \\
\hline 3 & 1.1 & 21 & 24 & $\mathrm{SiO}_{2}{ }^{\mathrm{b}}$ & 85 \\
\hline 4 & 1.1 & 21 & 24 & $\mathrm{Al}_{2} \mathrm{O}_{3}{ }^{\mathrm{c}}$ & 83 \\
\hline 6 & 1.2 & 21 & 24 & None & $80^{c}$ \\
\hline 7 & 1.0 & Reflux & 6 & None & 91 \\
\hline 8 & 1.1 & Reflux & 6 & None & 93 \\
\hline 9 & 1.1 & Reflux & 6 & $\mathrm{SiO}_{2}{ }^{\mathrm{b}}$ & 90 \\
\hline 10 & 1.1 & Reflux & 6 & $\mathrm{Al}_{2} \mathrm{O}_{3}{ }^{\mathrm{c}}$ & 88 \\
\hline 11 & 1.2 & Reflux & 6 & None & $83^{d}$ \\
\hline 12 & 1.3 & Reflux & 6 & None & $75^{\mathrm{e}}$ \\
\hline
\end{tabular}

${ }^{\mathrm{a}}$ Isolated yield. ${ }^{\mathrm{b}} 1$ gram; 40 microns, 32-63 mesh. ${ }^{\mathrm{c}} 1$ gram; neutral, 80-200 mesh.

$\mathrm{d}_{\mathrm{a}}$ trace amount of benzoic acid was observed. ${ }^{\mathrm{e}}$ approximately $8 \%$ benzoic acid was observed.

Having understood the optimal conditions for the oxidation, we proceeded to oxidize a wide variety of primary and secondary alcohols. The results of this part of the study are summarized in Table 2. The oxidation of benzyl alcohol based substrates oxidized quite efficiently; however, the powerful electron withdrawing $-\mathrm{NO}_{2}$ group severely inhibited the reaction as 1.7 molar equiv of 1 and a 36 hour reaction time were required to obtain a modest $62 \%$ yield (Table 2, entry 11). Benzyl alcohols containing substituents on the aromatic ring appeared to have no significant effect on the oxidation, however, 2- and 4-hydroxy benzaldehyde were obtained in a slightly lower yield most likely due to their increased water solubility (Table 2, entries 4 and 9). The presence of substituents on the benzylic carbon (Table 2, entries 12, 13, and 16-18) appeared to inhibit the oxidation and required slightly longer reactions times. Bulky sterically hindered groups (Table 2, entries 16-18) seemed to be the most pronounced when located on the benzylic carbon. Allylic alcohols (Table 2, entries 14-15) provided good yields at reasonable reaction times. Aliphatic based alcohols generally required significantly longer reactions times and also 
resulted in lower yields either due to their products' slight solubility in water (Table 2, entries 20-22) or to the presence of a congested and rigid bicyclic system (Table 2, entry 23).

Table 2. Aqueous oxidation of various alcohols in the presence of $\mathbf{1}$

\begin{tabular}{|c|c|c|c|c|}
\hline Entry & Substrate & Product & Time(hrs) & $\%$ Yield $^{\mathrm{a}}$ \\
\hline 1 & D & & 6 & 93 \\
\hline 2 & & & 6 & 88 \\
\hline 3 & & & 6 & 87 \\
\hline 4 & & & 6 & 77 \\
\hline 5 & & & 6 & 84 \\
\hline 6 & & & 6 & 85 \\
\hline 7 & & & 6 & 89 \\
\hline 8 & & & 6 & 85 \\
\hline 9 & & & 6 & 80 \\
\hline 10 & & & 6 & 84 \\
\hline
\end{tabular}


Table 2. Continued.

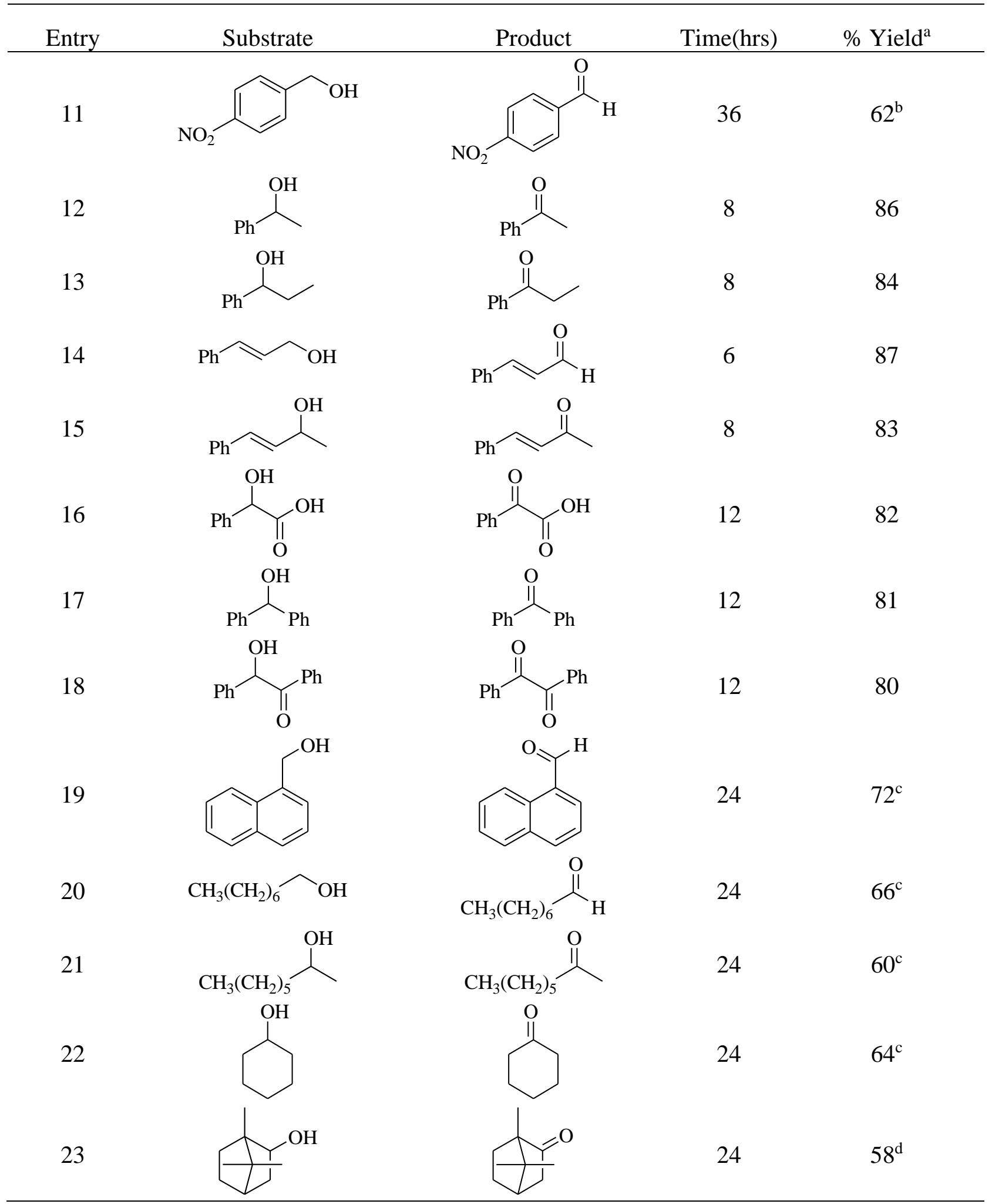

a Isolated yields. ${ }^{\mathrm{b}} 1.7$ molar equiv of 1 was required. ${ }^{\mathrm{c}} 1.4$ molar equiv of 1 was required. d2.0 molar equiv of $\mathbf{1}$ was required. 


\section{Conclusion}

We have developed an environmentally friendly method for the oxidation of a diverse group of primary and secondary aliphatic, primary allylic, and primary and secondary benzylic alcohols in the presence of $\mathbf{1}$ amid or devoid of catalyst (silica gel or alumina) in an aqueous media. Indeed, these 23 examples demonstrate that this general oxidative method appears to be quite functional group tolerant. Currently, we are further investigating the oxidation of primary and secondary aliphatic, substituted benzylic, and rigid alcohol systems to determine the limitations of this methodology.

\section{Experimental Section}

General. All chemicals were purchased from Fisher Scientific (except 4-amino-2,2,6,6tetramethyl piperidine; Sigma Aldrich) and were used as received. Melting points were obtained on a Fisher MelTemp ${ }^{\circledR}$ apparatus and are uncorrected. Gas chromatography-mass spectrometry was performed on a Thermo Scientific Trace DSQ II equipped with a Restek Rxi-5ms 30-m x 0.32-mm ID x $0.25 \mu \mathrm{m}$ df Crossbond $^{\circledR} 5 \%$ diphenyl:95\% dimethyl polysiloxane fused-silica glass capillary column. Silica gel (40 microns, 32-63 mesh) for flash chromatography ${ }^{8}$ was obtained from Scientific Adsorbents, Inc. Thin layer chromatograms were performed on Analtech Silica Gel GF plates (250 microns) and were visualized by UV. ${ }^{1} \mathrm{H}$ and ${ }^{13} \mathrm{C}$ NMR spectra were obtained on a Bruker DRX-400 spectrometer using $\mathrm{CDCl}_{3}$ as solvent. Proton spectra were referenced at $\delta=0$ for internal tetramethylsilane while carbon spectra were referenced at $\delta=77.2$ for ${ }^{13} \mathrm{CDCl}_{3}$.

\section{Preparation of 4-acetylamino-2,2,6,6-tetramethylpiperidine-1-oxoammonium tetrafluoro- borate (1)}

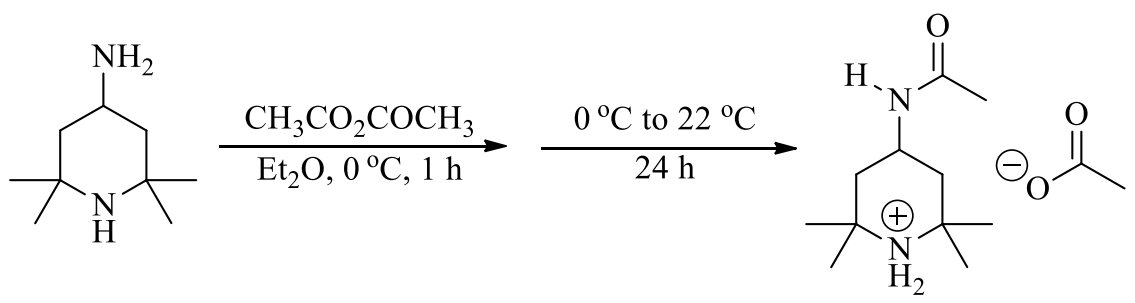

4-Acetylamino-2,2,6,6-tetramethyl-1-piperidinium acetate. The acetate was prepared following the procedure described by Bobbitt with slight modification. ${ }^{2 f}$ Thus, a solution of $220.5 \mathrm{~g}(0.853 \mathrm{~mol})$ of 4-amino-2,2,6,6-tetramethylpipeiridne in $525 \mathrm{~mL}$ of diethyl ether was cooled to $0{ }^{\circ} \mathrm{C}$ and a solution of $280.0 \mathrm{~mL}(2.96 \mathrm{~mol})$ of acetic anhydride in $150 \mathrm{~mL}$ of diethyl ether was then added dropwise for $2 \mathrm{~h}$. Upon complete addition, the reaction mixture was 
allowed to stir for $24 \mathrm{~h}$ at room temperature. The white slurry was vacuum filtered and washed with two 100-mL portions of diethyl ether and dried to constant weight in a fume hood to afford $230.5 \mathrm{~g}(92 \%)$ of a white solid which was used without purification: ${ }^{3 \mathrm{~b}} \mathrm{mp}=169-172{ }^{\circ} \mathrm{C}\left(\right.$ lit. $^{3 \mathrm{~b}}$ $\left.175^{\circ} \mathrm{C}\right)$.

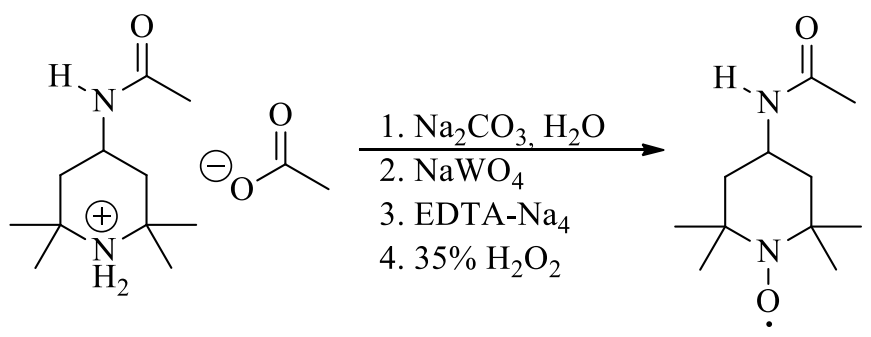

4-Acetylamino-2,2,6,6-tetramethyl-1-piperidinyloxy. The free radical was prepared following the procedure described by Bobbitt with slight modification. ${ }^{2 \mathrm{f}}$ Thus, in a $3 \mathrm{~L}$ beaker, a solution of $220.5 \mathrm{~g}(0.853 \mathrm{~mol})$ of 4-acetylamino-2,2,6,6-tetramethyl-1-piperidinium acetate in $1.55 \mathrm{~L}$ of distilled water was made basic by the addition of $153.8 \mathrm{~g}(1.45 \mathrm{~mol})$ of anhydrous sodium carbonate in small portions. The resulting white slurry was treated with $15.5 \mathrm{~g}$ (47.0 mmol) of sodium tungstate followed by $14.6 \mathrm{~g}(38.0 \mathrm{mmol})$ of ethylenediaminetetraacetic acid tetrasodium salt and was allowed to stir for $15 \mathrm{~min}$. To the reaction mixture, five $52-\mathrm{mL}$ portions of $35 \%$ hydrogen peroxide $(2.64 \mathrm{~mol}$ ) were added in $30 \mathrm{~min}$ intervals (during the additions, foaming can occur so caution must be observed). Upon complete addition, the orange slurry was allowed to stir for three days. The product mixture was then cooled to $0^{\circ} \mathrm{C}$ and filtered and the orange solid was washed once with $100 \mathrm{~mL}$ of ice-cold water. The filtrate and washing were combined and evaporated to approximately half the original volume, cooled to $0{ }^{\circ} \mathrm{C}$, and a second crop of crystals were filtered and washed with $25 \mathrm{~mL}$ of ice-cold water. The two crops of crystals were combined to give $172.1 \mathrm{~g}(95 \%)$ of an orange solid: ${ }^{3 \mathrm{~b}} \mathrm{mp}=145-146{ }^{\circ} \mathrm{C}$ (lit. $\left.{ }^{3 \mathrm{~b}} 147-148^{\circ} \mathrm{C}\right)$.

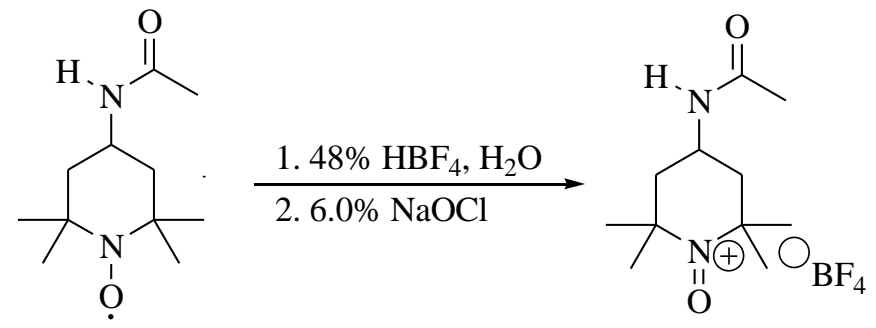

4-Acetylamino-2,2,6,6-tetramethylpiperidine-1-oxoammonium tetrafluoroborate (1). The oxoammonium salt was prepared following the procedure described by Bobbitt and Merbough. ${ }^{5}$ Thus, a slurry of $125.0 \mathrm{~g}(0.586 \mathrm{~mol})$ of 4-acetylamino-2,2,6,6-tetramethyl-1-piperidinyloxy and $245 \mathrm{~mL}$ of distilled water was treated by the slow dropwise addition $(\sim 30 \mathrm{~min})$ of $88.5 \mathrm{~mL}$ $(0.674 \mathrm{~mol})$ of a $48 \%$ aqueous solution of tetrafluoroboric acid followed by the slow dropwise addition $(\sim 3 \mathrm{~h})$ of $370.0 \mathrm{~mL}(0.322 \mathrm{~mol})$ of a $6.00 \%$ solution of sodium hypochlorite 
(commercial bleach, Clorox). Upon complete addition, the solution was allowed to stir for $24 \mathrm{~h}$ and was then cooled in an ice bath. The product mixture was vacuum filtered and washed with two 100-mL portions of ice cold saturated sodium bicarbonate, $100 \mathrm{~mL}$ of ice cold water, and $100 \mathrm{~mL}$ of diethyl ether. The filter cake was allowed to dry to constant weight to give $161.3 \mathrm{~g}$ (92\%) of a bright yellow solid. ${ }^{5} \mathrm{mp}=181-182^{\circ} \mathrm{C}$ (lit. $\left.{ }^{5} 184-185^{\circ} \mathrm{C}\right)$.

\section{General oxidation procedure}

A 100-mL round-bottomed flask equipped with a 1" magnetic stir bar was charged with the alcohol (10.0 mmol), oxoammonium salt $1,(11.0 \mathrm{mmol})$, and $25 \mathrm{~mL}$ of distilled water. The reaction mixture was vigorously stirred at reflux and monitored by thin-layer or gaschromatography in 2 to 12 -hour intervals by extracting a small aliquot with diethyl ether. The product mixture was extracted with three $25-\mathrm{mL}$ portions of diethyl ether. The combined organic extracts were washed with $10 \mathrm{~mL}$ of saturated sodium bicarbonate, $10 \mathrm{~mL}$ of brine, dried $\left(\mathrm{MgSO}_{4}\right)$, filtered, and concentrated by rotary evaporation. The resulting residue was filtered through a small pad of silica gel using 95:5 hexanes:ethyl acetate as eluent.

Spectral Data. All products prepared in this study are known compounds whose physical and spectroscopic properties are in accord with those reported in the literature.

Benzaldehyde (Table 2, entry 1). Colorless oil; ${ }^{1} \mathrm{H}$ NMR $\delta$ 7.51-7.55 (m, 2H), 7.61-7.65 (m, 1H), 7.87-7.90 (m, 2H), 10.02 (s, 1H); ${ }^{13} \mathrm{C}$ NMR $\delta 129.1,129.9,134.6,136.5,192.5$.

2-Methylbenzaldehyde (Table 2, entry 2). Colorless oil; ${ }^{1} \mathrm{H}$ NMR $\delta 2.58$ (s, 3H), $7.21-7.42$ $(\mathrm{m}, 3 \mathrm{H}), 7.75-7.77(\mathrm{~m}, 1 \mathrm{H}), 10.21(\mathrm{~s}, 1 \mathrm{H}) ;{ }^{13} \mathrm{C}$ NMR $\delta 19.6,126.6,132.01,132.2,133.9,134.5$, 140.6, 193.0.

2-Chlorobenzaldehyde (Table 2, entry 3 ). Colorless oil; ${ }^{1} \mathrm{H}$ NMR $\delta$ 7.34-7.39 (m, 1H) 7.42-7.44 $(\mathrm{m}, 1 \mathrm{H}) 7.49-7.53(\mathrm{~m}, 1 \mathrm{H}) 7.90(\mathrm{~d}, J 7.78 \mathrm{~Hz}, 1 \mathrm{H}) 10.46(\mathrm{~s}, 1 \mathrm{H}) ;{ }^{13} \mathrm{C}$ NMR $\delta 127.4,129.5$, $130.7,132.6,135.2,138.0,189.9$.

2-Hydroxybenzaldehyde (Table 2, entry 4). Colorless oil; ${ }^{1} \mathrm{H}$ NMR $\delta 2.16$ (br s, 1H), $6.98-$ $7.04(\mathrm{~m}, 2 \mathrm{H}), 7.51-7.57$ (m, 2H), 9.90 (s, 1H); ${ }^{13} \mathrm{C}$ NMR $\delta 117.8,120.1,133.9,137.2,196.8$.

2-Methoxybenzaldehyde (Table 2, entry 5). Colorless oil; ${ }^{1} \mathrm{H}$ NMR $\delta 3.82$ (s, 3H), $6.87-7.07$ $(\mathrm{m}, 2 \mathrm{H}), 7.49-7.52(\mathrm{~m}, 1 \mathrm{H}), 7.77-7.82(\mathrm{~m}, 1 \mathrm{H}), 10.41(\mathrm{~s}, 1 \mathrm{H}) ;{ }^{13} \mathrm{C}$ NMR $\delta 55.6,111.7,120.8$, 125.0, 128.4, 136.1, 162.0, 189.8 .

3-Methylbenzaldehyde (Table 2, entry 6). Colorless oil; ${ }^{1} \mathrm{H}$ NMR $\delta 2.44$ (s, 3H), $7.42-7.45$ $(\mathrm{m}, 3 \mathrm{H}), 7.67-7.70$ (m 1H), 9.99 (s, 1H); ${ }^{13} \mathrm{C}$ NMR $\delta 21.4,127.46,129.1,130.2,135.5,136.7$, 139.2, 192.8 .

4-Methylbenzaldehyde (Table 2, entry 7). Colorless oil; ${ }^{1} \mathrm{H}$ NMR $\delta 2.41$ (s, 3H) 7.38-7.45 (m, 2H) 7.64-7.68 (m, 2H) 9.97 (s, 1H); ${ }^{13} \mathrm{C}$ NMR $\delta 21.1,127.1,128.8,129.9,135.2,192.5$.

4-Bromobenzaldehyde (Table 2, entry 8). Pale yellow powder; ${ }^{1} \mathrm{H}$ NMR $\delta 7.69(\mathrm{~d}, J 8.31 \mathrm{~Hz}$, 2H), $7.75(\mathrm{~d}, J 8.31 \mathrm{~Hz}, 2 \mathrm{H}), 9.98(\mathrm{~s}, 1 \mathrm{H}) ;{ }^{13} \mathrm{C} \mathrm{NMR} \delta 130.0,131.2,132.7,135.3,191.3$; mp 53$55^{\circ} \mathrm{C}$ (lit. $\left.{ }^{9} 55-57^{\circ} \mathrm{C}\right)$. 
4-Hydroxybenzaldehyde (Table 2, entry 9). Pale yellow powder; ${ }^{1} \mathrm{H}$ NMR $\delta 4.92$ (br s, $1 \mathrm{H}$ ), $6.92(\mathrm{~d}, J 4.52 \mathrm{~Hz}, 2 \mathrm{H}), 7.77$ (d, $J 4.52 \mathrm{~Hz}, 2 \mathrm{H}), 9.76(\mathrm{~s}, 1 \mathrm{H}) ;{ }^{13} \mathrm{C}$ NMR $\delta 114.2,115.2,127.4$, 131.8, 191.2; mp 114-116 ${ }^{\circ} \mathrm{C}$ (lit. $\left.{ }^{10} 117^{\circ} \mathrm{C}\right)$.

4-Methoxybenzaldehyde (Table 2 , entry 10). Colorless oil; ${ }^{1} \mathrm{H}$ NMR $\delta 3.83$ (s, 3H) 6.96 (d, $J$ $8.74 \mathrm{~Hz}, 2 \mathrm{H}) 7.79$ (d, $J 8.74 \mathrm{~Hz}, 2 \mathrm{H}) 9.84(\mathrm{~s}, 1 \mathrm{H}) ;{ }^{13} \mathrm{C}$ NMR $\delta$ 55.6, 114.3, 130.0, 132.0, 164.6, 190.8 .

4-Nitrobenzaldehyde (Table 2, entry 11). Pale yellow solid; ${ }^{1} \mathrm{H}$ NMR $\delta 7.71$ (d, $J 4.24 \mathrm{~Hz}, 2 \mathrm{H}$ ), 8.21 (d, $J 4.24 \mathrm{~Hz}, 2 \mathrm{H}), 10.13(\mathrm{~s}, 1 \mathrm{H}){ }^{13} \mathrm{C}$ NMR $\delta$ 122.2, 123.2, 126.4, 129.5, 190.4; mp 101$102{ }^{\circ} \mathrm{C}$ (lit. $\left.{ }^{11} 104-105^{\circ} \mathrm{C}\right)$.

1-Phenylethanone (Table 2, entry 12). Colorless oil; ${ }^{1} \mathrm{H}$ NMR $\delta 2.54(\mathrm{~s}, 3 \mathrm{H})$ 7.38-7.43 (m, 2H) 7.47-7.53 (m, 1H) 7.89-7.92 (m, 2H); ${ }^{13} \mathrm{C}$ NMR $\delta 26.4,128.1,128.4,132.9,136.9,197.9$.

1-Phenyl-1-propanone (Table 2, entry 13). Colorless oil; ${ }^{1} \mathrm{H}$ NMR $\delta 1.20(\mathrm{t}, J 7.21 \mathrm{~Hz}, 3 \mathrm{H})$ $2.96(\mathrm{q}, J 7.21 \mathrm{~Hz}, 2 \mathrm{H}) 7.39-7.44(\mathrm{~m}, 2 \mathrm{H}) 7.48-7.53(\mathrm{~m}, 1 \mathrm{H}) 7.92-7.95(\mathrm{~m}, 2 \mathrm{H}) ;{ }^{13} \mathrm{C}$ NMR $\delta 8.0$, $31.5,127.7,128.3,132.6,136.7,200.5$.

(E)-3-Phenylpropenal (Table 2, entry 14). Pale yellow oil; ${ }^{1} \mathrm{H}$ NMR $\delta 6.71(\mathrm{t}, J 10.43 \mathrm{~Hz}, 1 \mathrm{H}$ ) 7.38-7.59 (m, 6H) $9.70(\mathrm{~d}, J 10.43,1 \mathrm{H}) ;{ }^{13} \mathrm{C}$ NMR $\delta 128.4,128.5,129.0,131.1,133.9,152.6$, 193.5 .

(E)-4-Phenyl-3-buten-2-one (Table 2, entry 15). Pale yellow needles; ${ }^{1} \mathrm{H}$ NMR $\delta 2.37$ (s, 3H), $6.72(\mathrm{~d}, J 16.28 \mathrm{~Hz}, 1 \mathrm{H}), 7.38-7.41(\mathrm{~m}, 3 \mathrm{H}), 7.50-7.55(\mathrm{~m}, 3 \mathrm{H}) \quad{ }^{13} \mathrm{C} \mathrm{NMR} \delta 27.2,126.9$, 128.1, 128.8, 130.3, 134.2, 143.1, 198.0; mp 37-39 ${ }^{\circ} \mathrm{C}$ (lit. $\left.{ }^{12} 40-42{ }^{\circ} \mathrm{C}\right)$.

Benzoylformic acid (Table 2, entry 16). White solid; ${ }^{1} \mathrm{H}$ NMR $\delta 7.52-7.56(\mathrm{~m}, 2 \mathrm{H}), 7.69-$ $7.73(\mathrm{~m}, 1 \mathrm{H}), 8.28-8.31(\mathrm{~m}, 2 \mathrm{H}), 10.08(\mathrm{~s}, 1 \mathrm{H}) ;{ }^{13} \mathrm{C}$ NMR $\delta 129.2,131.3,131.89,135.9,163.1$, 184.7; mp 63-64 ${ }^{\circ} \mathrm{C}$ (lit. ${ }^{13}$ 64-65 ${ }^{\circ} \mathrm{C}$ ).

Benzophenone (Table 2, entry 17). White solid; ${ }^{1} \mathrm{H}$ NMR $\delta 7.46-7.51(\mathrm{~m}, 4 \mathrm{H}), 7.57-7.62(\mathrm{~m}$, 2H), 7.80 - $7.82(\mathrm{~m}, 4 \mathrm{H}) ;{ }^{13} \mathrm{C}$ NMR $\delta$ 128.5, 130.3, 132.6, 137.8, 196.9; mp 46-47 ${ }^{\circ} \mathrm{C}$ (lit. ${ }^{14} 47-$ $\left.48{ }^{\circ} \mathrm{C}\right)$.

Benzil (Table 2, entry 18). Off-white Solid; ${ }^{1} \mathrm{H}$ NMR $\delta 7.51-7.54$ (m, 4H), $7.64-7.69$ (m, 2H), 7.97 - 7.99 (m, 4H); ${ }^{13} \mathrm{C}$ NMR $\delta 129.2,130.1,133.2$, 135.1, 194.8; mp 92-94 ${ }^{\circ} \mathrm{C}$ (lit. ${ }^{15} 94-95$ $\left.{ }^{\circ} \mathrm{C}\right)$.

1-Naphthaldehyde (Table 2, entry 19). Pale yellow oil; ${ }^{1} \mathrm{H}$ NMR $\delta 7.57-7.60(\mathrm{~m}, 2 \mathrm{H}), 7.67-$ $7.71(\mathrm{~m}, 1 \mathrm{H}), 7.91-7.93(\mathrm{~m}, 1 \mathrm{H}), 7.97-7.98(\mathrm{~m}, 1 \mathrm{H}), 8.09$ (d, $J 8.22 \mathrm{~Hz}, 1 \mathrm{H}), 9.25-9.27$ (d, $J$ $8.52 \mathrm{~Hz}, 1 \mathrm{H}), 10.40$ (s, 1H) ${ }^{13} \mathrm{C}$ NMR $\delta 125.0$ (2 C's), 127.1, 128.7, 129.2, 130.7, 131.5, 133.9, $135.5,136.8,193.7$.

Octanal (Table 2, entry 20). Colorless oil; ${ }^{1} \mathrm{H}$ NMR $\delta 0.89$ (t, $\left.J 7.00 \mathrm{~Hz}, 3 \mathrm{H}\right)$ 1.29-1.31 (m, 8H) $1.62(\mathrm{t}, J 7.00 \mathrm{~Hz}, 2 \mathrm{H}) 9.77(\mathrm{~s}, 1 \mathrm{H}) ;{ }^{13} \mathrm{C} \mathrm{NMR} \delta 14.2,22.3,22.8,29.2,29.3,31.8,44.1,203.0$.

2-Octanone (Table 2, entry 21). Colorless oil; ${ }^{1} \mathrm{H}$ NMR $\delta 0.868$ (t, $\left.J 7.03 \mathrm{~Hz}, 3 \mathrm{H}\right), 1.23-1.29$ $(\mathrm{m}, 6 \mathrm{H}), 1.54(\mathrm{p}, J 3.92 \mathrm{~Hz}, 2 \mathrm{H}), 2.13(\mathrm{~s}, 3 \mathrm{H}), 2.39$ (t, J $7.40 \mathrm{~Hz}, 2 \mathrm{H}) ;{ }^{13} \mathrm{C}$ NMR $\delta 14.2,22.7$, 24.0, 29.0, 31.8, 44.0, 209.5.

Cyclohexanone (Table 2, entry 22). Colorless oil; ${ }^{1} \mathrm{H}$ NMR $\delta 1.72$ (p, J $\left.6.30 \mathrm{~Hz}, 2 \mathrm{H}\right) 1.86$ (p, $J$ $6.30 \mathrm{~Hz}, 4 \mathrm{H}) 2.33(\mathrm{t}, J 6.30 \mathrm{~Hz}, 4 \mathrm{H}) ;{ }^{13} \mathrm{C} \mathrm{NMR} \delta 25.2,27.2,42.13,212.2$. 
Camphor (Table 2, entry 23). White solid; ${ }^{1} \mathrm{H}$ NMR $\delta 0.82$ (s, 3H), 0.90 (s, 3H), 0.943 (s, 3H), $1.29-1.42$ (m, $2 \mathrm{H}), 1.63-1.69(\mathrm{~m}, 1 \mathrm{H}), 1.85-1.92(\mathrm{~m}, 2 \mathrm{H}), 2.08$ (t, J 4.52 Hz, $1 \mathrm{H}), 2.30-$ $2.37(\mathrm{~m}, 1 \mathrm{H}) ;{ }^{13} \mathrm{C}$ NMR $\delta 9.5,19.3,20.0,27.2,30.0,43.2,43.4,46.9,57.8,219.6$; mp 170-173 ${ }^{\circ} \mathrm{C}$ (lit. $\left.{ }^{16} 173-174{ }^{\circ} \mathrm{C}\right)$.

\section{Acknowledgements}

This project was partially funded by the University of Pittsburgh at Greensburg and by a grant from the University of Pittsburgh's Central Research Development Fund. We thank Professors James M. Bobbitt and William F. Bailey from the University of Connecticut for their valuable insight into this study. We also graciously thank Mr. Daniel Terwilliger and Mr. Michael Mercadante from the University of Connecticut for completing the NMR analysis of our samples.

\section{References}

1. For a recent review, see Merbouh, N.; Bobbitt, J. M.; Brückner, C. Org. Prep. \& Proc. Int. 2004, 36, 1.

2. For select preparations of various oxoammonium salts, see (a) Zhdanov, R. I.; Golubev, V. A.; Rozantsev, É. G. Izv. Akad. Nauk USSR., Ser. Khim. 1970, 1, 188; Chem. Abstr. 1970, 72, 111227. (b) Golubev, V. A.; Zhadov, R. I.; Rozantsev, E. G. Izv. Akad. Nauk USSR., Ser. Khim. 1970, 1, 186. Chem. Abstr. 1970, 72, 111229. (c) Golubev, V. A.; Zhdanov, R. I.; Gida, V. M.; Rozantsev, E. G. Izv. Akad. Nauk USSR., Ser. Khim. 1971, 4, 768; Chem. Abstr. 1971, 75, 63571. (d) Kagiya, T.; Komuro, C.; Sakano, K.; Nishimoto, S. Chem. Lett., 1983, 365. (e) Miyazawa, T.; Endo, T.; Shiihashi, S.; Okawara, M. J. Org. Chem. 1985, 50, 1332. (f) Bobbitt, J. M. J. Org. Chem. 1998, 63, 9367.

3. For select oxidations, see ref 2f. (a) Bobbitt, J. M.; Flores, M. C. L. Heterocycles 1988, 27, 509. (b) Ma, Z.; Bobbitt, J. M. J. Org. Chem. 1991, 56, 6110. (c) de Nooy, A. E. J.; Besemer, A. C.; van Bekkum, H. Synthesis 1996, 1153. (d) Kernag, C. A.; Bobbitt, J. M.; McGrath, D. V. J. Tetrahedron Lett. 1999, 40, 1635.

4. (a) Bobbitt, J. M. Chem. Eng. News 1999, 77, 6. (b) Molecules 1999, 4, M102.

5. Bobbitt, J. M.; Merbough, N. Organic Syntheses; Wiley: New York, 2005, Vol. 82; p 80.

6. For recent reviews, see (a) Li, C.-J.; Chen, L. Chem. Soc. Rev. 2006, 1, 68. (b) Minikata, S.; Komatsu, M. Chem. Rev. 2009, 109, 711. (c) Chanda, A.; Foken, V. V. Chem. Rev. 2009, 109, 724.

7. Breslow, R. Acc. Chem. Res. 2004, 37, 471.

8. Fair, J. D.; Kormos, C. M. J. Chromatog. A 2008, 1211, 49.

9. Coleman, G. H.; Honeywell, G. E. Organic Syntheses; Wiley: New York, 1959; Vol. 2, p 89. 
10. Buramoy, A.; Markowitsch-Buramoy, I. J. Chem. Soc. 1936, 36.

11. Brown, H. C.; McFarlin, R. F. J. Am. Chem. Soc. 1956, 78, 252.

12. Drake, N. L.; Allen, P. Organic Syntheses; Wiley: New York, 1941; Coll. Vol. 1, p 77.

13. Claisen, L. Chem. Ber. 1877, 10, 429.

14. Marvel, C. S.; Sperry, W. M. Organic Syntheses; Wiley: New York, 1941; Coll. Vol. 1, p 95.

15. Clark, H. T.; Dreger, E. E. Organic Syntheses; Wiley: New York, 1941; Coll. Vol. 1, p 87.

16. Ritter, J. J. J. Org. Chem. 1933, 55, 3322. 